\title{
BALANCE DE LA LUCHA ANTITUBERCULOSĂ EN EL ECUADOR
}

\section{I1. SJTUACION ACTUAL}

Dr. ARRMANDO PAREJA CORONEL

Presidente de L.E.A., Guaytquil

\section{LA MORBILIDAD POR 'TUBERCULOSIS}

Fn la acturdidiud, el problema de la uttherulosis on el Ecuados aúr es comm plcjo cumo en ta meyor parte de kss pueblos del mund $($, a pesar de que lins grandes ciudactes cuentan con elementos de hecha crtganizada y se ha podido

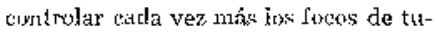
bercuiosin por rredjo de los exámenes gistemúlicos de los contactos, habiém. duse iniciado yis en la ciudad do Guayaquil ia flumioperpilaxis soeundaria.

La frecuencia de la traberculasis en las grandes ciudades, según nuestras estid'sticas, ha disminuido Rigeramente: en cambio la unda infecciosa, por circunstancias obvias, se hu propagidiy o intensificado en los campus $y$ el problema primordial, en la actualidar, es la acción prevendiva-profilóntica en las zunas rurales y el martenimierto de equipos mó

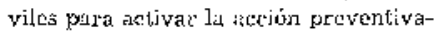
curativa del eornpexsino, Je1 misma que so hầe dia d día mís impreseindjble, deseruciadamento la capuctirhad económica no perruite efectivizar osta acción en debida forma.

T'ABLA I

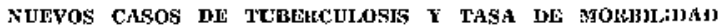
POR NUEYOS CASOS

\begin{tabular}{|c|c|c|c|c|}
\hline ANCS & THE. PUIKHONAR & 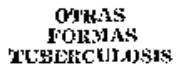 & TO'PAI & $\begin{array}{c}\text { TASA PUK } \\
\text { TAOB.HU KIAB. }\end{array}$ \\
\hline 1956 & 4.351 & 115 & 4.466 & $1.14,9$ \\
\hline 1957 & 4.662 & 37 & 4.699 & 117,6 \\
\hline .1458 & 5.402 & 61 & 5.463 & 132,4 \\
\hline 1859 & 4.699 & 23 & 4.692 & 1.11 .1 . \\
\hline 1960 & 4.981 & 19 & 5.000 & $, 11,5,3$ \\
\hline 1901 & 5.638 & 22 & 5.600 & $127,1$. \\
\hline 1962 & $5.0 \mathrm{se}$ & 33 & 5.115 & $i(t 9,9$ \\
\hline
\end{tabular}


La Tabla I de Lil inciderieia general de la tubercujosis que se inserts, puede dar llevar a la falsa conthusión de que dicha incidencia se mantiene mis o monss en el mismo nivel descle el año 1956 a 1962 . Estu se tebe a que intervictren los fucclouses de lo propugación de lu andia tuberculosa hacia lus canpos un los cuales la incidencia es mayot: er contraste a la disminuśón en Ias cindades.

\section{IrA MORTALIDAD HOR TUBERCULOSIS}

En cuanto a la reırbalidad, cl cuadro generat de la nación no da ni aproxinadimente la realidad de las defuncimes pur una a olria rnfermedad como la tuberculosis, pues on likn zonas rurales més del $60 \%$ de las defunciones no llewan certificatos medicos; cxtoncos el roferie ol ísdice de mortalidad

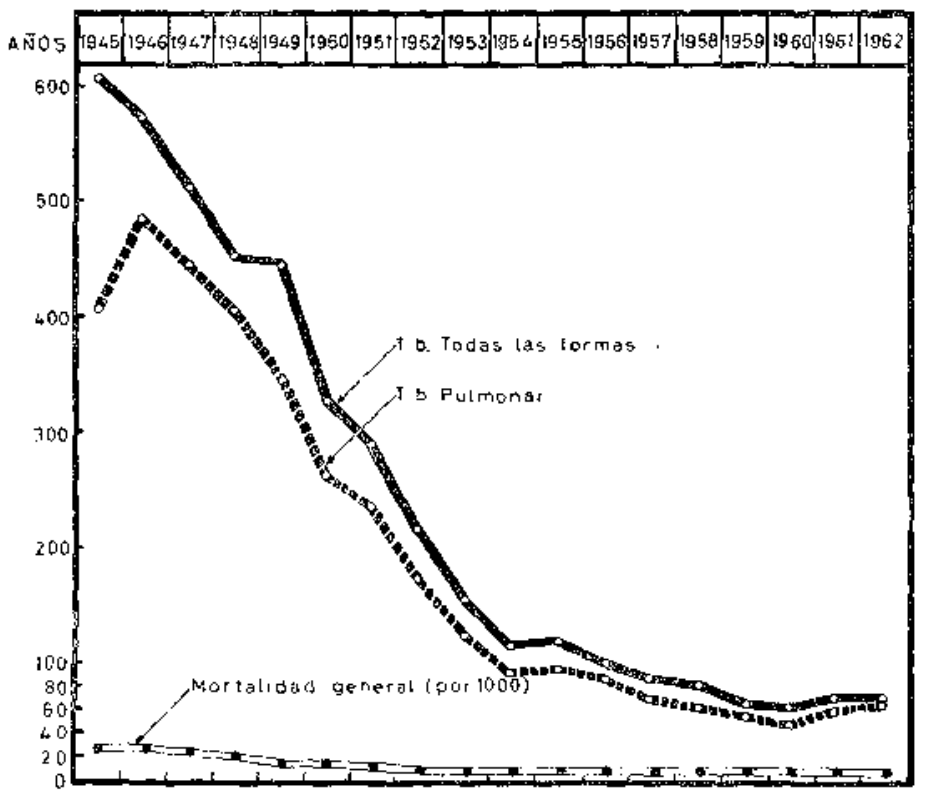

Fig. 1.-WYortalidad por tuberenlosis ell la ciedad di: Guyaquil-DL tasa do mortialjilad

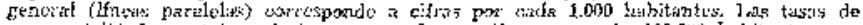

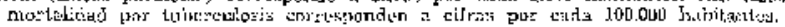




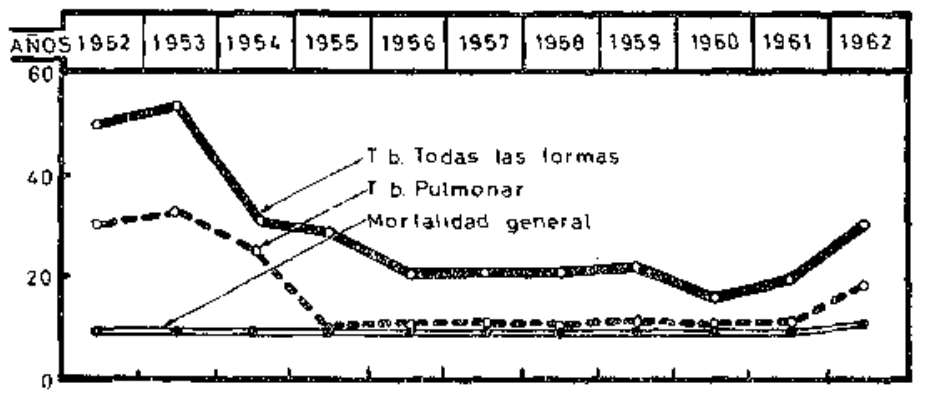

Fig. 2.-Mortulidul por tuberculosis su in ciudad de Quito,-Io tosa do mortzlidad gene-

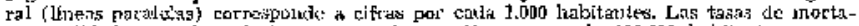

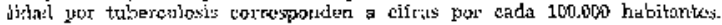

a la totalidad de la población de la Repriblica, tendríamos un íncice sumunconte bajo, no compatible con Ia reatidad patológioa y únicamente los indices do rnortelidad de las grindes ciudades y potblaciones mayores de 10.000 habitantes son las más aproximados a lo roulided y en ellos se anoda чл espectacular descenso do la mortalidad, conn puede apreciarse lá curva de las defunciones registradas en la ciudad de Guayuquil, de 1945 a 1962 (Fig. 1) la que efectivannonte es fiel reflejo de lu paIpable disminución de la mortadidad en la ciudad de Guayuquil, donde se cncuentra la mavor parte di los elementos de lucha, pues a la morbitidad propia de la ciudad se añate le procedente de zonas rurales, aún de las más lejarias, que sun atendidas en los Dispensarios "Arosemena" y "Mato Martinez", que tienen u' mixino movimirnto de steneiones en eI yails.
El descemso de mortalidadi, por turlrertulosis, ha sido especialmerite notorio hasia 1.954 , año en el que se Ifegó it una țasa de aproximadamente 1/1.000. De 1954 a 1960 la mortalidad ha continuado disoninuyendo muy ljgreraments, para aumentar, aldugue th forma escusa en los siguientes ûnos.

In cuanto a la ciudad de Quito (Fig. 2), en gerieral la tasa de mortalidat es inferior a la de Guayaquil. Cuando se estudia detenidamente las estadíslicas, se aprecia que esta dismimución cn la tasa de mortalidad, se refiere principalnente a mortalidad infartil y juverit.

Isa tasa de mortialidad por tubertulosis ha sido simpre más byja que en

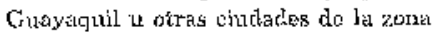
litoral del país. También en Quito la mortasidad por tubereulisis descenctio espetialmente hasta 1954, luego su disminución ha sido muy lenta hasta 
1960, para repuntar un ligeru aumento en los qunos siguienics.

Fistas dos muestrus, correspondientes a las dos ciudades más propulosás ctel país purden lintuarse como ejemplos de in que ha sucedido con la mortalidad por tuberculusis en las ciudades. En cuanto a las zoras rurales, en doncle nús bion ha habido ondas de exacerbasión de lá tuberculoris, as probabic que tembién lin morlalidad haya aumentado. Lesdo luego, no se dispurzon de estadistcas fehacientes, subro dichas yonas durales.

\section{BIDLIOGRAFIA}

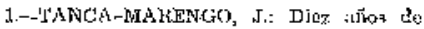
suctsa antituberculosin on cil Lucuador, yitss. 10, 19, 31, 26, 39, 41, 44; 1940-1950).

2.-PAITEJA CORORFL, A : Informe salual dc) Cornité Ejccutivo Re Lìn Ecuatoriano Arstitubrectulosa, Abril 4, 1!62, วád: 15-18.

3.-PAREJA-COPONE,L, A.; Informe alsual del Conmize lajecutivo de Liga Yountorians Autluborculosit, Felyrexo 25, J903, páts. 19-Ist.

4.--EALTIA-COINONEI, A: Lr) forme del Comblit Fiecutivo de Ligya Jeriatroticusta

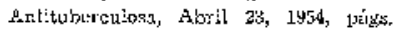
8-9 y unexos.
5.-PAREJA-CORONEL, A.: Jríme munl

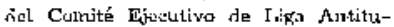
bexculosa, Agnato 11, 2055, Jáipa 6-t1.

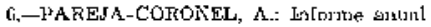
del Coturté s'jevertivo de Jiga beutioriani Anlitubseulosa, Marzo 21, 1956. púgs. $2-3 \mathrm{y}$ ancosos

7.-PAIBEIA-CORONEL, A.: Informe arut del Conité bsecutivo de Esera Eeuater.spa histutubertulast, Fenrerd 21, 1957. pésss. 9, 10 y $11 \mathrm{y}$ onexos.

8. HARETh-CORONEL, A.: Infonne anual del Comité Ejoculivo de Liga Ecuatu-

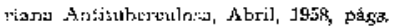
$38,22,23$.

a. -..PAREJA-CORONKL, $A$,; Irforme snual del Comillé Ejccutivo de Ligar Eeuatoriana Antituberrulosi1, Abrid, 1959, páge.

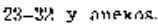

10-PAIRJA-CORUNEL, A.: Infarme anual del Comité Sijecutivo de Liga Ecusto.

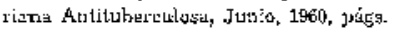
22-2d y tatexos.

11. JALESA-CORONEI, A.: Informa : del Comite Fjecuílvo de Liga Eojiznrigns Antitllbercu'ose, Noviembic, I961, prígs. 20-21 y snexos.

12.-JIJON, M. A.: Informe anual del Comitc .Fjestitivo de Lija Ecushariana Ant:iutrorculosa, Nơięnbre, 1861, págs. 20-21 Y rTivets.

13. JIJON, M. A.: Iniorme anual del Comi1é Ejecutivo de Jigga Ecuutoriana Antitu-

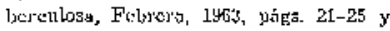
inexos. 